\title{
Análise prototípica das representações sociais sobre as infecções sexualmente transmissíveis entre adolescentes
}

\section{Social representations related to sexually transmitted diseases in adolescentes. Prototypical analysis} \section{(1) PSicOCGENTE}

ARTíCULO DE
INVESTIGACIÓN
Copyright $\odot 2018$
by Psicogente

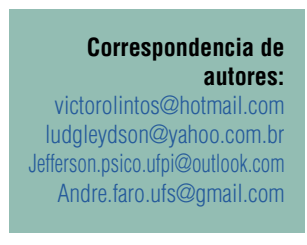

Recibido: 08-11-17 Aceptado: 05-06-18 Publicado: 08-01-19

\section{Análisis prototípico de las representaciones sociales sobre las infecciones sexualmente transmisibles entre adolescentes}

José Victor de Oliveira Santos (iD - Ludgleydson Fernandes de Araújo iD
Jefferson Luiz De Cerqueira Castro (iD
Universidade Federal do Piauí, Parnaíba, Brasil
André Faro iD
Universidade Federal de Sergipe, Aracaju, Brasil

Resumo

Objetivo: Este estudo teve como objetivo apreender e analisar as representações sociais de adolescentes acerca das doenças sexualmente transmissíveis.

Método: Participaram 576 adolescentes, com idade média de 15,67 anos ( $D P=1,66)$. Utilizou-se a técnica de associação livre de palavras, com o estímulo indutor "infecções sexualmente transmissíveis", que foi utilizada a técnica da análise prototípica, através do programa IRAMUTEQ.

Resultados: As representações sociais dos participantes enfatizam os riscos e as formas de prevenção, os conhecimentos básicos sobre as infecções sexualmente transmissíveis, a irresponsabilidade de fazerem sexo desprotegido mesmo sabendo das consequências.

Conclusão: Identifica-se a necessidade de implantação de programas de educação em saúde dentro das escolas, utilizando métodos didáticos e reflexivos com profissionais da saúde, que permitam a mudança de atitudes diante da exposição as infecções sexualmente transmissíveis.

Palavras-chave: Infecções sexualmente transmissíveis, Representações sociais, Adolescentes.

Abstract

Objective: This study aims to figure out and analyze social representations related to sexually transmitted diseases in adolescents.

Method: 576 adolescents, mean age 15,67 years $(S D=1,66)$ were sampled. The free word association technique and the inductor stimulus "sexually transmitted infections", used as prototypical analysis technique, through the IRAMUTEQ program.

Results: Participants' social representations emphasize on risks and preventive ways, a basic knowledge related to sexually transmitted infections, irresponsibility of having unprotected sex even knowing the consequences.

Conclusions: The need to implement health education programs within schools, using didactic and reflexive methods with health professionals that make possible the change of behaviors towards exposure to sexually transmitted infections.

Keywords: Sexually transmitted infections, Social Representations, Adolescents. 
Resumen

Objetivo: Aprehender y analizar las representaciones sociales de adolescentes acerca de las enfermedades sexualmente transmisibles.

Método: Participaron 576 adolescentes, con edad media de 15 años ( $D P=1,66)$. Se utilizó la técnica de asociación libre de palabras, con el estímulo inductor "Infecciones Sexualmente Transmisibles", y la técnica del análisis prototípico, a través del programa IRAMUTEQ.

Resultados: Las representaciones sociales de los participantes enfatizan los riesgos y las formas de prevención, los conocimientos básicos sobre las infecciones sexualmente transmisibles, la irresponsabilidad de hacer sexo desprotegido aun sabiendo de las consecuencias.

Conclusión: Se identifica la necesidad de implantación de programas de educación en salud dentro de las escuelas, utilizando métodos didácticos y reflexivos con profesionales de la salud, que permitan el cambio de actitudes ante la exposición a las infecciones sexualmente transmisibles.

Palabras clave: Enfermedades de transmisión sexual, Representaciones sociales, Adolescentes.

\section{INTRODUÇÃO}

Dentre alguns problemas de saúde pública presentes no Brasil, a prevalência de Infecç̧ões Sexualmente Transmissíveis (IST) é um deles, mesmo com o investimento em prevenção, diagnóstico, tratamento e educação em saúde (Costa, Araújo, Araújo, Gubert, \& Vieira, 2015; Santos et al., 2018). Segundo o Ministério da Saúde (2014), pessoas entre 15 e 24 anos apresentam maior número de ocorrências nos dados sobre IST. Além da AIDS, a doença inflamatória pélvica, a sífilis, gonorreia, hepatites virais, lifogranuloma venéreo, tricomoníase, cancro mole, donovanose, herpes genital e condiloma acuminado são IST presentes nos índices epidemiológicos (Ministério da Saúde, 2014).

De acordo com a Organização Mundial de Saúde (2010), as doenças mais comuns a partir das estatísticas anuais são: Clamídia 1.967.200, gonorreia 1.541.800, sífilis 937.000, HPV 685.400 e herpes genital 640.900. As principais causas da transmissão das IST são a não utilização do preservativo, idade precoce, múltiplos parceiros sexuais e práticas sexuais de risco, que em muitos casos resultam também em gravidez (Bermúdez, Araújo, Reyes, Hernández-Quero, \& Teva, 2016; Teva, Bermúdez \& Ramiro 2013). Para promover informação acerca do tema, o Ministério da Saúde publicou um manual de bolso sobre IST, que aborda minunciosamente aspectos que vão desde as estratégias de prevenção até o diagnóstico e manejo dos procedimentos a serem seguidos pelo sujeito infectado (Ministério da Saúde, 2006).

Destaca-se um estudo recente que aborda a baixa escolaridade, relações desiguais de gênero e o pouco contato dos profissionais da saúde com adolescentes são fatores contribuintes para a prevalência de IST entre as mulheres (Sampaio, Santos, Callou \& Souza, 2011). Neste direcionamento, 
Costa et al., (2015) enfatizam a importância de protagonizar os adolescentes na elaboração de métodos para discussões acerca das IST. Afinal, na adolescência a maioria das pessoas vivenciam suas primeiras experiências sexuais. Com isso, abordar alguns métodos de cuidado primário evita as doenças e ainda uma gravidez indesejada. Outros autores abordam que os cursos de graduação devem implementar ações que problematizem o tema, para que a atuação profissional seja pautada em dados científicos e eficazes (Almeida et al., 2015).

Em estudo prévio recente com adolescentes de 14 anos, viu-se que a maioria dos participantes sabem o que são IST, porém a escola não fornece atividades para aprofundar a aprendizagem (Rampelotto, Höner, Oliveira, Bottega \& Santos, 2016). Não só estudos produzidos no Brasil mostram dados semelhantes. Teva et al. (2013) complementam elucidando a importância de medidas psicossociais como método para redução do número de pessoas infectadas no contexto de adolescentes espanhóis. Destaca-se ainda, que o percentual de jovens que conseguem ingressar no ensino superior se mostra mais responsáveis quanto ao uso de preservativo e métodos anticonceptivos (Dessunti \& Reis, 2012), o que necessita estudos futuros a respeito.

Uma pesquisa prévia buscou compreender os fatores de risco e proteção a partir da ótica de adolescentes (Mesquita, Costa, Luna, Silva, \& Pinheiro, 2017), sendo que foram encontrados resultados semelhantes aos riscos apontados nos estudos supracitados (Teva et al., 2013; Sampaio et al., 2011; Santos et al., 2018). Porém, especificamente quanto aos fatores protetivos, identificou-se o parceiro sexual fixo e confiável e uma boa relação familiar, além de amizades e até professores que possibilitam o diálogo sobre IST tendem a minimizar a exposição ao adoecimento (Mesquita et al., 2017). Assim, destaca-se que a inserção de medidas psicossociais a respeito das IST por meio de uma rede de apoio que envolve desde familiares até os profissionais tende a fornecer subsídios para a redução de comportamentos sexuais de risco. Com isso, julga-se relevante a continuidade de pesquisas que busquem identificar conhecimentos da vida cotidiana acerca das IST junto à população adolescente.

Neste estudo empregou-se como suporte teórico a teoria das representações sociais que reproduz discursos elaborados no senso comum, que coletivamente tornam-se científicos, pois representam socialmente a aprendizagem de uma população, neste sentido, as representações sociais podem ser utili- 
zadas como um instrumento para apreender os conhecimentos sobre determinado fenômeno. As representações sociais, como forma de nomear e dar significado aos aspectos cotidianos, envolvem desde a percepção até a formação de atitudes, isto é, é uma perspectiva teórica que contempla as maneiras de se posicionar diante de algum objeto (Jodelet, 2001).

A abordagem estrutural explica que as representações sociais não surgem apenas de um processo cognitivo ou de subjetivação objetal (Campos \& Rouquette, 2003). No entanto, Jean Claude Abric apresentou esta nova ótica de formação representacional, a qual aborda a representação como algo organizado e estruturado, que atravessa diversas dimensões psicossociais (Abric, 1998; Abric, 2002). O núcleo central executa três funções: a de gerar, organizar e estabilizar as representações sociais (Abric \& Campos, 1996; Crusoé, 2004), sendo que para Abric (1998) as representações sociais são "um conjunto de informações, de crenças, de opiniões e de atitudes sobre um objeto dado" (p.19). Portanto, o núcleo central apresenta uma representação social ao perpassar pelas três funções anteriormente citadas, sendo ele o mais rígido a mudanças. Noutras palavras, havendo mudança no núcleo central, há mudança nas representações sociais de modo mais amplo (Crusoé, 2004). Além do núcleo central existe o sistema periférico, que possui a característica de ser mais facilmente modificado, pois geralmente está associado a vivências cotidianas (Abric, 1998).

Por fim, entende-se que a realização deste estudo contribui social e academicamente com a temática, na medida em que há uma escassez de estudos a respeito, bem como poderá acessar o conhecimento destes adolescentes acerca das formas de prevenção, diagnóstico e tratamento das IST. Diante do exposto, o presente estudo objetivou identificar e analisar as representações sociais dos adolescentes acerca das IST.

\section{MÉTODO}

\subsection{Desenho do estudo}

Trata-se de um estudo qualitativo, ex post facto, descritivo e de recorte transversal.

\subsection{Participantes}

Contou-se com a participação de 576 adolescentes de dois estados brasileiros, Piauí e Sergipe. A idade média foi de 15,7 anos (DP=1,66), com 57,1\% 
do gênero feminino e $42,9 \%$ masculino. Todos os participantes cursavam o ensino médio. Quanto a religiosidade, 38,5\% se declaram católicos, 25,3\% evangélicos, 2,1\% espíritas, 6,4\% de outras religiosidades. Além desses, $27,75 \%$ disseram não seguir nenhuma religião. Em relação a amizades com pessoas que já tiveram ou têm alguma IST, $74,6 \%$ não conhecem ninguém e $25,4 \%$ têm amizades.

\subsection{Instrumentos}

Utilizou-se questionário sociodemográfico para caracterização dos participantes, com perguntas como: Idade, sexo, religiosidade, amizade com pessoas com IST. Além disso, para apreender as representações sociais acerca das IST, a sigla foi utilizada como estímulo e cada participante evocou as cinco primeiras palavras que vieram à mente, de acordo com a Técnica de Associação Livre de Palavras - TALP que possibilita uma análise a luz da teoria das representações sociais, tendo em vista que as evocações explicam o fenômeno pesquisado (Nóbrega \& Coutinho, 2003).

\subsection{Procedimentos de coleta de dados}

O presente estudo foi aprovado pelo Comitê de Ética em Pesquisa da Universidade Federal de Sergipe, com o CAAE 54236016.0.0000.5546. Os critérios da investigação seguiram os princípios éticos para pesquisas com seres humanos conforme o proposto pelo Conselho Nacional de Saúde na resolução no 510/2016. Após a aprovação, iniciou-se a coleta de dados, no qual era apresentado um Termo de Consentimento Institucional - TCl nas escolas com ensino médio, em seguida, para os participantes, o Termo de Consentimento Livre e Esclarecido. Neles estavam explícitos que a pesquisa era de cunho acadêmico, com o anonimato garantido e que os participantes poderiam se negar a participar a qualquer momento da pesquisa. $\mathrm{O}$ formulário levou cerca de 15 minutos para ser respondido.

\subsection{Procedimentos de análise de dados}

A estatística descritiva dos dados sociodemográficos foram obtidas por meio do programa estatístico IBM SPSS 23. Além dessa análise, conduziu-se a análise prototípica, que é realizada a partir da técnica de evocações de palavras com base em algum termo indutor, e mediante isto, organiza-se os dados a partir da frequência e da Ordem Média de Evocação (OME). Para a realização desta análise, é criado o banco de dados no Calc do Open Office, 
que é executado no programa IRAMUTEQ e que compila arquivos textuais a partir do software $\mathrm{R}$ (Team, 2013). O resultado da análise possibilita a visualização de quatro zonas: a primeira referente ao núcleo central, a segunda com o sistema periférico, a terceira com elementos periféricos contrastantes e a último também com dados periféricos. Nessas zonas, exibem-se as palavras juntamente com a frequência de aparição das respostas e OME, que significa a posição média em que a palavra apareceu na classificação de ordem das evocações (Wachelke \& Wolter, 2011).

\subsection{Conflito de interesse}

Não houve conflito de interesse entre os pesquisadores, tendo em vista que todos possuíam os mesmos objetivos com o presente estudo.

\section{RESULTADOS}

A análise prototípica das representações sociais consiste na organização das palavras evocadas a partir da frequência e da ordem média de evocações, e mediante os valores de cada palavra, elas ficam divididas em quatro quadrantes (Vergès, 1992). Os critérios para cada quadrante serão explicados juntamente com os resultados encontrados nas representações sociais das infecções sexualmente transmissíveis.

Foram inseridas 554 linhas de comando, contendo a frequência das palavras evocadas e sua ordem média de evocação (OME). Os elementos centrais correspondem as palavras com maior frequência e uma ordem de evocação alta, isto é, palavras que foram as primeiras a serem evocadas, a principal palavra foi "doença $(f=112 ; O M E=2,4)$ ", seguida de "cuidado $(f=101 ; O M E=2,4)$ " e "camisinha $(f=84 ; O M E=2,4)$ ". Identifica-se que a parte mais primitiva das representações sociais já integra tanto a doença, quanto a prevenção e tratamento, isto é, é possível afirmar que o adolescente já tem uma noção básica do tema. A próxima palavra evocada foi "Aids $(f=75 ; O M E=2,0)$ ", que é a doença sexualmente transmissível que mais traz impactos biopsicossociais na vida dos sujeitos (Teva et al., 2013), sendo, também, a IST mais conhecida.

Na visualização da Tabela 1 é possível identificar que os elementos que compõem o núcleo central possuem o OME menor que 2,84, e os da primeira periferia maior que 2,84 , e para estarem nesses dois primeiros quadrantes, a frequência de evocações foi acima do valor de 10,81. 
Tabela 1

Elementos centrais e primeira periferia das representações sociais sobre IST

\begin{tabular}{|c|c|c|c|c|c|c|}
\hline & \multicolumn{3}{|c|}{ ORDEM MÉDIA DE EVOCAÇÃO (OME) <2,84 } & \multicolumn{3}{|c|}{ ORDEM MÉDIA DE EVOCAÇÃO (OME) >2,8 } \\
\hline & \multicolumn{3}{|c|}{ ELEMENTOS CENTRAIS } & \multicolumn{3}{|c|}{ ELEMENTOS DA PRIMEIRA PERIFERIA } \\
\hline & PALAVRA & $\mathbf{F}$ & OME & PALAVRA & $\mathbf{F}$ & OME \\
\hline & Doença & 112 & 2,4 & Sexo & 184 & 2,9 \\
\hline & Cuidado & 101 & 2,4 & Tratamento & 74 & 3,1 \\
\hline & Camisinha & 84 & 2,4 & Morte & 52 & 3,6 \\
\hline & Aids & 75 & 2,0 & Gonorreia & 46 & 3,1 \\
\hline & Prevenção & 59 & 2,4 & Sífilis & 44 & 3,0 \\
\hline & Descuido & 53 & 2,4 & Preconceito & 32 & 3,3 \\
\hline & Medo & 38 & 2,8 & Contato & 29 & 3,0 \\
\hline & Perigoso & 35 & 2,7 & Herpes & 28 & 3,7 \\
\hline & Vírus & 27 & 2,0 & Transmissíveis & 28 & 3,0 \\
\hline & Proteção & 21 & 2,5 & Relação & 26 & 2,9 \\
\hline \multirow[t]{11}{*}{$\begin{array}{c}\text { Frequência } \geq \\
10,81\end{array}$} & Irresponsabilidade & 19 & 2,4 & Medicação & 25 & 3,1 \\
\hline & Risco & 18 & 2,8 & HPV & 19 & 3,2 \\
\hline & Contagioso & 18 & 2,4 & Atenção & 16 & 3,6 \\
\hline & Transmissível & 17 & 2,5 & Dor & 14 & 3,1 \\
\hline & Preservativo & 13 & 2,7 & HIV & 14 & 3,3 \\
\hline & Sifilis & 13 & 2,8 & Preocupação & 14 & 3,1 \\
\hline & Saúde & 12 & 2,5 & Tristeza & 13 & 3,1 \\
\hline & Contaminação & 12 & 2,2 & Sofrimento & 12 & 3,2 \\
\hline & Ruim & 11 & 2,5 & Homem & 12 & 3,7 \\
\hline & & & & Responsabilidade & 12 & 2,9 \\
\hline & & & & Mulher & 11 & 3,8 \\
\hline
\end{tabular}


Outras palavras evocadas foram: "prevenção $(f=59 ; O M E=2,4)$ ", "descuido $(f=53$; $O M E=2,4)$ ", “medo $(f=38 ; O M E=2,8)$ ", "perigoso $(f=35 ; O M E=2,7)$ ", "vírus $(f=27 ; O M E=2,0)$ ". Tais vocábulos permitem visualizar que as representações sociais partem dos fatores de risco e proteção, ou seja, o entendimento em que o participante tem acerca de agravamentos futuros e como se proteger disto. Outras evocações menos frequentes, mas que se situaram no núcleo das representações sociais foram: "proteção $(f=21 ; O M E=2,5)$ ", "irresponsabilidade $(f=19 ; O M E=2,4)$ ", "risco $(f=18 ; O M E=2,8)$ ", "contagioso $(f=18$; $O M E=2,4)$ ", "transmissível $(f=17 ; O M E=2,5)$ ".

Na primeira periferia, que remete aos conteúdos que reforçam e dão sustentabilidade às crenças encontradas no núcleo central, são palavras muito frequentes e com ordem de evocação mediana, as representações sociais apontam a palavra "sexo $(f=184 ; O M E=2,9)$ ", como a mais evocada, seguida das palavras: tratamento $(f=74 ; O M E=3,1)$, morte $(f=52 ; O M E=3,6)$, gonorreia $(f=46 ; O M E=3,1)$, sífilis $(f=44 ; O M E=3,0)$, preconceito $(f=32 ; O M E=3,3)$, contato $(f=29$; $O M E=3,0)$, herpes $(f=28 ; O M E=3,7)$ e transmissíveis $(f=28$; $O M E=3,0)$. Quanto às representações sociais periféricas, percebe-se que o adolescente tem conhecimento sobre algumas das IST, e tem entendimento de que a morte e o preconceito são possíveis causas. As representações sociais periféricas são construtos mais atualizados e que tendem a se modificar de acordo com mudanças sociais (Abric, 1998), ou seja, são mais flexíveis que o núcleo central. Diante disto, as palavras "contato $(f=29 ; O M E=3,0)$ ", "sofrimento $(f=12 ; O M E=3,2)$ " e "tristeza $(f=13 ; O M E=3,1)$ " sugerem questionamentos atuais sobre que tipo de contato leva a transmissão de IST e que além da afetação biológica, as emoções negativas podem surgir.

Os elementos contrastantes, que tiveram pouca frequência de evocação, mas uma ordem de evocação alta, e na segunda periferia, evocações com baixa frequência e ordem de evocação baixa (Wachelke \& Wolter, 2011). Esses dois quadrantes, representam dados que foram menos evocados, porém trazem peculiaridades das representações sociais que são significativas para a compreensão na análise prototípica. Na tabela 2, é possível visualizar as palavras evocadas, a partir da frequência e OME. 
Tabela 2

Elementos contrastantes e segunda periferia das representações sociais sobre IST

\begin{tabular}{|c|c|c|c|c|c|c|}
\hline & \multicolumn{3}{|c|}{ ORDEM MÉDIA DE EVOCAÇÃO (OME) <2,84 } & \multicolumn{3}{|c|}{ ORDEM MÉDIA DE EVOCAÇÃO (OME) >2,84 } \\
\hline & \multicolumn{3}{|c|}{ ELEMENTOS CONTRASTANTES } & \multicolumn{3}{|c|}{ ELEMENTOS DA SEGUNDA PERIFERIA } \\
\hline & PALAVRA & $\mathbf{F}$ & OME & PALAVRA & $\mathbf{F}$ & OME \\
\hline \multirow{19}{*}{$\begin{array}{c}<10,81 \\
\text { Frequência }\end{array}$} & Conhecimento & 10 & 2,7 & Informação & 10 & 2,9 \\
\hline & Relação Sexual & 7 & 2,0 & Hospital & 10 & 3,6 \\
\hline & Vergonha & 7 & 2,7 & Cura & 10 & 3,3 \\
\hline & Segurança & 6 & 2,8 & Problema & 10 & 3,7 \\
\hline & Desespero & 6 & 2,8 & Consciência & 9 & 2,9 \\
\hline & Contaminação & 6 & 2,8 & Herpes genital & 8 & 3,8 \\
\hline & Prevenir & 6 & 2,8 & Grave & 8 & 3,4 \\
\hline & Sexualmente & 5 & 2,5 & Desproteção & 8 & 3,2 \\
\hline & Atenção & 5 & 2,6 & Infecção & 7 & 3,0 \\
\hline & Confiança & 5 & 2,8 & Vida & 7 & 3,6 \\
\hline & Remédio & 5 & 2,4 & Sangue & 5 & 3,6 \\
\hline & Consequência & 5 & 2,4 & Raiva & 5 & 3,6 \\
\hline & Ajuda & 5 & 2,4 & Incurável & 5 & 3,8 \\
\hline & Câncer & 4 & 1,8 & Preocupação & 5 & 4,0 \\
\hline & Festa & 4 & 2,5 & Insegurança & 5 & 4,0 \\
\hline & Superação & 4 & 1,5 & Triste & 5 & 3,8 \\
\hline & Jovem & 4 & 2,8 & Prejudicial & 5 & 4,0 \\
\hline & Ter cuidado & 4 & 2,8 & Higiene & 5 & 3,4 \\
\hline & Incômodo & 3 & 2,3 & Condiloma & 4 & 4,5 \\
\hline \multirow{4}{*}{$\begin{array}{c}<10,81 \\
\text { Frequênci }\end{array}$} & Horror & 3 & 2,3 & Incômodo & 4 & 3,5 \\
\hline & Falta de cuidado & 3 & 2,7 & Carnaval & 4 & 3,5 \\
\hline & Educação & 3 & 2,7 & Prostituição & 4 & 3,0 \\
\hline & Problema social & 2 & 2,5 & Isolamento & 4 & 4,2 \\
\hline
\end{tabular}

Os elementos contrastantes não apresentam representações sociais distantes das apresentadas nos outros quadrantes, porém a palavra "conhecimento $(f=10 ; O M E=2,7)$ ", "prevenir $(f=6 ; O M E=2,8)$ ", "atenção $(f=5 ; O M E=2,6)$ ", "ajuda $(f=5 ; O M E=2,4)$ " e "educação $(f=3 ; O M E=2,7)$ " evidenciam que em contraste as representações sociais baseadas nas doenças, nas causas e formas de adquirir a enfermidade, os adolescentes relatam algum conhecimento das formas de prevenção. Além disso, revelam que a "falta de cuidado $(f=3 ; O M E=2,7)$ " resulta em algo além do "incômodo $(f=3 ; O M E=2,3)$ " e da "consequência $(f=5 ; O M E=2,4)$ " da "contaminação $(f=6 ; O M E=2,8)$ "; isto é, estar infectado com alguma IST, é também um problema de ordem psicossocial, mas a educação surge como uma forma de ajuda que auxilia na manutenção de comportamentos saudáveis. A "vergonha $(f=7 ; O M E=2,7)$ " e o "desespero $(f=6 ; O M E=2,8)$ " são características comuns nas pessoas que já foram infectados por IST. 
Os termos presentes na segunda periferia das representações sociais complementam os apresentados na primeira periferia, porém, com uma OME e frequência menor (Wachelke \& Wolter, 2011). Como pode ser visualizado no quadro 2, apresenta-se dois tipos de IST "Herpes genital $(f=8 ; O M E=3,8)$ " e "Condiloma $(f=4 ; O M E=4,5)$ ", e ainda a palavra "informação $(f=10$; $O M E=2,9)$ ", "hospital ( $f=10 ; O M E=3,6)$ ", "cura $(f=10 ; O M E=3,3)$ " e "higiene $(f=5 ; O M E=3,4)$ ". Pode-se entender que estas seriam representações sociais direcionadas ao cuidado. Os termos "problema", "grave", "raiva", "incurável", "triste", "prejudicial" e "isolamento" surgem no sentido representacional negativo das consequências advindas a partir da infecção com alguma IST. Outro fato a ser demonstrado é o termo "carnaval $(f=5 ; O M E=3,4)$ ", que se associa ao estigma da época em que o contágio das IST é mais suscetível, por se tratar de um evento há uma integração populacional e cultural (Francisco et al., 2015).

Discute-se aqui, então, a medida adequada para que os sujeitos tenham relações sexuais seguras, tendo em vista que nas representações sociais dos sujeitos desta pesquisa, os mesmos entendem como se dá os processos, desde a prevenção até o tratamento. Logo, percebe-se que não basta conhecer. É fundamental que haja algum tipo de ativação comportamental para que os sujeitos passem a fazer uso do preservativo, em qualquer situação (Araújo, Lôbo, Santos \& Sampaio, 2017).

\section{DISCUSSÃO}

Os dados deste estudo explanam que os adolescentes possuem conhecimentos básicos sobre as IST, o fato é que não basta conhecer, há a necessidade de praticar os métodos de prevenção. Um estudo sobre atitudes e práticas sobre as IST descreve que as práticas sexuais seguras nem sempre são utilizadas (Santos et al., 2018). Outro estudo aborda sobre a relação de confiança entre casais para o não uso do preservativo, justificando que o uso do mesmo atrapalha na obtenção do prazer (Sousa, Silva, \& Palmeira, 2014). Encontrou-se no trabalho de Costa et al. (2016), realizado com jovens universitárias, o medo sentido quando o ato sexual é feito sem proteção e explicam que ter parceiro sexual fixo faz com que se reduza o uso do preservativo, e além destes, os participantes da pesquisa afirmam que as vezes esquecem ou acham incômodo usar. Os autores abordaram ainda sobre vulnerabilidade em que os participantes viam como vulneráveis as profissionais do sexo e pessoas sem informação, esse achado também foi documentado no estudo de Mesquita et al. (2017). 
Além dos adolescentes, estudos mostram sobre vulnerabilidades em outros grupos. Como idosos (Silva et al., 2018), pescadores de comunidades ribeirinhas (Ribeiro et al., 2017), Pessoas com deficiência visual (Lima, 2016), pessoas com deficiência física (Aragão et al., 2016). Porém, o fato é que os adolescentes estão em maior número, no que se diz a vulnerabilidades frente as IST, e um dos motivos é a necessidade de descoberta de algo que é tido como um tabu, o sexo (Almeida et al., 2017).

Amoras, Campos e Beserra (2015) dissertam sobre os fatores que contribuem para a vulnerabilidade de adolescentes diante das IST, como "o início da vida sexual precoce, falta de informação referente a realização do ato sexual, não utilização do preservativo, desigualdade de gênero, baixa renda e vulnerabilidade social" (Amoras, Campos \& Beserra, 2015, p.166). Os autores enfatizam a desigualdade de gênero associando o cuidado à figura feminina, e ainda, trazem que devido a mulher engravidar, ela que deve apresentar o preservativo durante a relação sexual.

Outro estudo demonstra que os adolescentes veem o uso de preservativo como um método contraceptivo, e não como proteção para possíveis infecções do ato sexual (Junqueira et al., 2016). A distribuição gratuita de preservativo viabiliza o uso do mesmo (Thiesen, 2018), porém é necessário que a adesão ao preservativo seja por toda a população.

A partir da necessidade de proteção, os adolescentes exprimem que o risco do contágio advém da irresponsabilidade, que muitas vezes os levam ao ato sexual sem o uso de preservativo. Um estudo aborda que os homens preferem não usar camisinha e em muitos casos obrigam suas parceiras sexuais a terem relação sexual sem proteção (Sampaio et al., 2011). A partir dos dados deste estudo, infere-se que as representações sociais negativas auxiliam na percepção da necessidade de cuidado, no sentido de que, por ser algo que traz consequências ruins, a cautela é essencial na prevenção.

No ensino médio, fase que se intensifica as vivências sexuais, os livros pouco relatam sobre IST e HIV/Aids, abordam a parte da biológica da contaminação e não se discute questões aprofundadas sobre prevenção e tratamento (Lopes \& Brandão, 2013). Este déficit na educação sexual pode ser o causador de comportamentos sexuais de risco (Silva, Jacob, \& Hirdes, 2017; Sá et al., 2015; Araújo et al., 2017). Ainda assim, um estudo evidencia que HIV/Aids, Sífilis e HPV são as IST mais conhecidas por adolescentes (Krabbe et al., 2016), dados semelhantes aos deste estudo. 
As representações sociais centrais revelam a síntese acerca dos danos, da necessidade de proteção e do uso do preservativo. Destarte, constata-se que os adolescentes têm conhecimento prévio acerca das IST. Este entendimento demonstra o que alguns estudos apontam, a exemplo da necessidade da execução de ações em saúde para informar sobre práticas sexuais saudáveis e manejo diante da transmissão (Rampelotto et al., 2016; Teva et al., 2013).

A palavra "responsabilidade" esteve entre as representações periféricas e seu antônimo nas representações centrais, esses termos demonstram que a prática sexual de risco não parte do desconhecimento acerca das IST e sim da ausência de responsabilidade, na perspectiva dos adolescentes que participarem do estudo. Mesmo que as pessoas saibam que o correto é usar preservativo, muitas vezes, movidos pelo desejo e busca do prazer, acabam negligenciando o uso (Santos et al., 2016). Em estudo prévio relacionado a este tema, observou-se que os jovens entendem que a transmissão vai além do sexo anal, oral e vaginal, acrescentando também transfusão de sangue, objetos cortantes contaminados, amamentação e outros (Costa et al., 2016).

O receio de sofrer preconceito é um fator que faz com que os sintomas físicos sejam ocultados e muitas vezes retardam o início do processo de tratamento, essa estigmatização do sujeito com IST pode causar diversos problemas psicológicos (Guevara-Sotelo \& Hoyos-Hernández, 2018; Henkes, Dias, \& Cruz, 2016). Um artigo com adolescentes que possuem o HIV a partir de infecção vertical, aponta o receio dos mesmos em buscarem ter relações com outros adolescentes e o medo do abandono após revelar sobre possuir o HIV (Sehnem, Pedro, Ressel \& Vasquez, 2018). Esse medo de rejeição é apontado em outro estudo (Mburu et al., 2014).

O contexto escolar se apresenta como um local apropriado para o desenvolvimento de ações em saúde visando informar sobre as infecções sexualmente transmissíveis. Estudos abordam que um dos métodos de cativar a atenção dos adolescentes é através de atividades lúdicas e educativas (Cunha et al., 2017; Oliveira, Queiroz, de Sousa Chaves, Branco, \& Mendes, 2017; Carneiro et al., 2015). Outro estudo sugere que a abordagem sobre as IST com base nos estereótipos compartilhados pela sociedade é uma forma de desconstruir e propagar dados de realidade (Guimarães et al., 2017). A sensibilização diante das consequências das IST auxilia na percepção e mudança 
das atitudes quanto aos métodos de prevenção (Silva \& Cortez, 2017; Oliveira \& Patel, 2015).

Diante dos achados deste estudo, foi possível identificar as representações sociais acerca das IST, em que as palavras evocadas na parte central se referem aos termos mais comuns acerca da problemática. As representações sociais periféricas registram assuntos menos abordados, mas que todo modo, algumas palavras evocadas semelhantes se fazem presente em todas as zonas, o que reflete o contexto representacional não apresenta separação, e sim, complementação. Conclui-se que as representações sociais das IST, estão associadas a um sentimento negativo, advindo das possíveis consequências de aquisição, e que os adolescentes possuem entendimento, além do senso comum, porém, na prática, muitas vezes são deixados a se levar pelo desejo sexual.

O desenvolvimento deste estudo, além de possibilitar documentar as representações sociais concernindo as IST, talvez também possa ter funcionado como um meio de intervenção, que fez com que adolescentes pudessem explanar seus pensamentos acerca deste tema que é emergente nesta fase do desenvolvimento. Os resultados sugerem que as representações sociais dos adolescentes são coerentes com os fatores de risco e proteção e ainda assim, muitos se arriscam.

Espera-se que os resultados aqui obtidos possam servir como escopo para futuras intervenções psicossociais que atuem de maneira a desenvolver atitudes mais saudáveis frente as IST. Tendo em vista que o método mais efetivo para combater as IST é a prevenção, faz-se necessário conscientizar a população em geral, o que pode ser iniciado já desde a infância e, obviamente, enfatizado na adolescência, na expectativa de que futuramente os índices de adoecimento em todos os grupos sociais sejam cada vez menores. Ressalta-se, assim, que não adianta conhecer os hábitos saudáveis, é importante serem utilizados na prática e compreender as representações sociais pode ser um passo nessa direção. 
Financiamento: O presente estudo foi aprovado pelo Comitê de Ética em Pesquisa da Universidade Federal de Sergipe com o CAAE: 54236016.0.0000.5546, e realizado em parceria com a Universidade Federal do Piauí.

\section{REFERÊNCIAS}

Abric, J. C. (1998). A abordagem estrutural das representações sociais. Em A. S. P. Moreira \& D. C. Oliveira (Orgs.), Estudos interdisciplinares de representação social (pp.27-38). Goiânia: AB.

Abric, J. C. (2002). L'approche structurale des représentations sociales: Développements récents. Psychologie et Société, 4, 81-103. Recuperado de: http://www. europhd.net/bibliographic-item/lapproche-structurale-des-repr\%C3\%A9sentations-sociales-d\%C3\%A9veloppements-r\%C3\%A9cents

Abric, J. C. \& Campos, P. H. F. (1996). Les éducateurs e leur représentation de l'enfant de rue au Brésil. Em J. C. Abric (Org.), Exclusion sociale, insertion ET prévention (pp.137-149). Saint-Agne: Erès.

Almeida, A. N. F. S., Silva, A. F., Correia, D. C. S., Sumaya, C. A. O., Miranda, W. F., Peixoto, A. L. V. A., \& Oliveira, R. D. C. B. (2015). Ações educativas para prevenção de gravidez indesejada e de doenças sexualmente transmissíveis (IST) entre universitários. Entre Aberta Revista de Extensão, 1(1), 1-1. Recuperado de: http:// revistas.cesmac.edu.br/index.php/entreaberta/article/view/299/218

Almeida, R. A. S., \& Corrêa, R. G. C. F., \& Rolim, I. T. P., \& Hora, J. M., \& Linard, A. G., \& Coutinho, N. P. S., \& Oliveira, P. S. (2017). Conhecimento de adolescentes relacionados às doenças sexualmente transmissíveis e gravidez. Revista Brasileira de Enfermagem, 70(5), 1087-1094. http://dx.doi.org/10.1590/0034-7167-20160531.

Amoras, B. C., Campos, A. R., \& Beserra, E. P. (2015). Reflexões sobre vulnerabilidade dos adolescentes a infecções sexualmente transmissíveis. PRACS: Revista Eletrônica de Humanidades do Curso de Ciências Sociais da UNIFAP, 8(1), 163-171. Recuperado de: https://periodicos.unifap.br/index.php/pracs/article/view/1668.

Aragão, J. S., França, I. S. X., Coura, A. S., Medeiros, M., Campos, C., \& Enders, B. C. (2016). Vulnerabilidade associada às infecções sexualmente transmissíveis em pessoas com deficiência física. Revista Ciência \& Saúde Coletiva, 21(10), 3143-3152. http://dx.doi.org/10.1590/1413-812320152110.20062016.

Araújo, L. F., Lôbo, C. J. C., Santos, J. V. O., \& Sampaio, A. V. F. C. (2017). Concepções Psicossociais acerca do Conhecimento sobre a AIDS das Pessoas que Vivem com o HIV. Revista Colombiana de Psicología, 26(2), 219- 230. http://dx.doi. org/10.15446/rcp.v26n2.59349

Bermúdez, M. D. L. P., Araújo, L. F. D., Reyes, A. O., Hernández-Quero, J., \& Teva, I. (2016). Analysis of cognitive variables and sexual risk behaviors among infected and HIV-uninfected people from Spain. AIDS care, 28(7), 890-897. http://dx.doi. org/10.1080/09540121.2016.1161163.

Campos, P. H. F., \& Rouquette, M. L. (2003). Abordagem estrutural e componente afetivo das representações sociais. Psicologia: reflexão e crítica, 16(3), 435-445. Recuperado de: http://www.scielo.br/pdf/\%0D/prc/v16n3/v16n3a03.pdf

Carneiro, R. F., Silva, N. C., Alves, T. A., Brito, D. C., \& Oliveira, L. L. (2015). Educação sexual na adolescência: uma abordagem no contexto escolar. SANARE-Revista de Políticas Públicas, 14(1),104-108. Recuperado de: https://sanare.emnuvens.com. br/sanare/article/view/617/334.

Costa, A. C. P. D. J., Araújo, M. F. M. D., Araújo, T. M. D., Gubert, F. D. A., \& Vieira, N. F. C. (2015). Protagonismo de adolescentes na prevenção de doenças 
sexualmente transmissíveis. Acta paul. Enferm, 28(5), 482-487. http://dx.doi. org/10.1590/1982-0194201500080

Costa, S. P., Silva, T. B., Rocha, T. A., Guisande, T. C. C. A., Cardoso, A. M., Gomes, J. L., \& Guisande, M. T. C. R. (2016). Saberes e representações de vulnerabilidade para IST/HIV/AIDS por jovens universitárias. REVISTA DE PSICOLOGIA, 10(31), 25-42. http://dx.doi.org/10.14295/idonline.v10i31.483

Crusoé, N. M. D. C. (2004). A Teoria das Representações Sociais em Moscovici e sua importância para a pesquisa em educação. Caderno de Filosofia e Psicologia da Educação, 2(2), 105-114. Recuperado de: http://www.uesb.br/editora/publicacoes/aprender/edicoes/Aprender\%20n.\%202.pdf\#page $=10$

Cunha, A. C. G. D. P., Caramelo, F., Patrício, M., Camarneiro, A. P., Cardoso, S. M., \& Pita, J. R. (2017). Impacto de um programa de intervenção educativa nos comportamentos sexuais de jovens universitários. Revista de Enfermagem Referência, (13), 71-82. Recuperado de: https://search.proquest.com/openview/56f9d3084a0e28bed437d240a6d4c799/1?pq-origsite=gscholar \&cbl=2036194.

Dessunti, E. M., \& Reis, A. O. A. (2012). Vulnerabilidade às IST/aids entre estudantes da saúde: estudo comparativo entre primeira e última série. Ciência, Cuidado e Saúde, 11(5), 274-283. http://dx.doi.org/10.4025/cienccuidsaude.v11i5.17738

Francisco, M. T. R., Fonte, V. R. F., Spindola, T., Martins, E. R. C., Costa, C. M. A., \& Pinheiro, C. D. O. P. (2015). Conhecimento sobre HIV/aids e a utilização do preservativo entre os participantes do carnaval. Revista Cubana de Enfermería, 30(3). Recuperado de: http://www.revenfermeria.sld.cu/index.php/enf/article/ view/295/94

Guevara-Sotelo, Y., \& Hoyos-Hernández, P. A. (2018). Vivir con VIH: experiencias de estigma sentido en personas con VIH. Psicogente, 21(39), 127-139. https://doi. org/10.17081/psico.21.39.2827

Guimarães, D. A., Oliveira, C. A. M., Lima, R. A., Silva, L. C., Avelar, C. R. T., \& Gama, C. A. P. (2017). Formação em saúde e extensão universitária: discutindo sexualidade e prevenção de IST/aids. Revista Brasileira de Pesquisa em Saúde/Brazilian Journal of Health Research, 19(2), 124-132. Recuperado de: http://www.periodicos.ufes.br/RBPS/article/viewFile/18870/12847

Henkes, R., Dias, J. M., \& Cruz, R. F. (2016). Considerações sobre a análise institucional em um serviço público de atendimento especializado a portadores de IST/ Aids. Trabalho apresentado em Salão de Ensino e de Extensão. Anais do Salão de Ensino e de Extensão, Recuperado de: http://online.unisc.br/acadnet/anais/ index.php/salao_ensino_extensao/article/view/15322.

Jodelet, D. (2001). As representações sociais. Rio de Janeiro: EDUERJ. Recuperado de: https://books.google.com.br/books/about/As_representa\%C3\%A7\%C3\%B5es_ sociais.html?id=zhWfAAAACAAJ\&redir_esc $=y$

Junqueira, M., Silva, E. V. B., Santos, J. R., Silva, P. P. B. A., Cavalcanti, I. F. M., \& Silva, F. P. (2016). Infecções sexualmente transmissíveis: atuação do enfermeiro nas ações educativas na adolescência. Revista Saúde-UNG, 10(1 ESP), 128. Recuperado de: http://revistas.ung.br/index.php/saude/article/view/2725.

Krabbe, E. C., Brum, M. D., Capeletti, C. P., Costa, T. D. S., Mello, M. L., Vieira, P. R., \& de Carvalho, T. G. M. L. (2016). Escola, sexualidade, práticas sexuais e vulnerabilidades para as infecções sexualmente transmissíveis (IST). Revista interdisciplinar de ensino, pesquisa e extensão, 4(1), 75-84. Recuperado de: http://www.revistaeletronica.unicruz.edu.br/index.php/eletronica/article/view/4387/pdf_73.

Lima, S. P. (2016). Infecções sexualmente transmissíveis e seus fatores de risco em pessoas com deficiência. Trabalho de Conclusão de Curso (Graduação em Enfer- 
magem), Universidade Estadual da Paraíba, Campina Grande. Recuperado de: http://dspace.bc.uepb.edu.br/jspui/handle/123456789/12848

Lopes, A. P. B., \& Brandão, G. O. (2013). A abordagem da AIDS nos livros de biologia. Brasília: UNICEUB. Recuperado de: http://repositorio.uniceub.br/ bitstream/235/6464/1/21052166.pdf

Mburu, G., Hodgson, I., Kalibala, S., Haamujompa, C., Cataldo, F., Lowenthal, E. D., \& Ross, D. (2014). Adolescent HIV disclosure in Zambia: barriers, facilitators and outcomes. Journal of the International AIDS Society, 17(1). http://dx.doi. org/10.7448/IAS.17.1.18866

Mesquita, J. S., Costa, M. I. F., Luna, I. T., Silva, A. A., \& Pinheiro, P. N. C. (2017). Fatores de risco e de proteção entre adolescentes em relação às IST/hiv/aids. Revista de enfermagem UFPE, 11(3), 1227-1233. https://doi.org/10.5205/19818963-v11i3a13498p1227-1233-2017

Ministério da Saúde (2006). Manual de bolso controle das doenças sexualmente transmissíveis - IST. Ministério da Saúde: Brasília. Recuperado de: http://bvsms. saude.gov.br/bvs/publicacoes/manual_controle_das_dst.pdf

Ministério da Saúde (2014). Boletim Epidemiológico: AIDS e IST 2014. Ministério da Saúde: Brasília. Recuperado de: http://portalarquivos2.saude.gov.br/images/ pdf/2014/dezembro/01/boletim-epidemiologico-hiv-aids-2014.pdf

Nóbrega, S. M. \& Coutinho, M. P. L. (2003). O Teste de Associação Livre de Palavras. In: M. P. L. Coutinho (Org.), Representações Sociais: Abordagem interdisciplinar. (pp.67-77). Editora Universitária, UFPB, João Pessoa. Recuperado de: https:// books.google.com.br/books/about/Representa\%C3\%A7\%C3\%B5es_sociais. html?id=DSrXAAAAMAAJ

Oliveira, F. A., de Queiroz, A. M., de Sousa Chaves, M. A., Branco, M. F. C. C., \& Mendes, I. C. (2017). Atividades lúdicas desenvolvidas com adolescentes escolares sobre prevenção de infecções sexualmente transmissíveis. Revista Interdisciplinar, 10(3), 53-63. Recuperado de: https://revistainterdisciplinar.uninovafapi.edu.br/ index.php/revinter/article/view/1124.

Oliveira, L. P., \& Patel, B. N. (2015). Programa de prevenção às infecções sexualmente transmissíveis e HIV/AIDS: uma experiência na Bahia. Revista Guará, (3), 113-117. https://doi.org/10.30712/guara.v3i3.9842

Organização Mundial de Saúde - OMS (2010). Relatório Mundial de saúde. Organização Mundial de Saúde. Recuperado de: http://www.who.int/eportuguese/ publications/WHR2010.pdf

Rampelotto, R. F., Hörner, R., Oliveira, F., Bottega, A. \& Santos, S. O. (2016). Educação em saúde na adolescência: uma experiência acadêmica com alunos de escola pública. Anais do Salão Internacional de Ensino, Pesquisa e Extensão, 7(3), recuperado de http://publicase.unipampa.edu.br/index.php/siepe/article/ view/15175/4801

Ribeiro, L. L., Moreira, W. C., de Carvalho, A. R. B., de Sousa, M. D. C. P., Carvalho, M. L., \& de Castro, T. M. B. Q. (2017). Vulnerabilidades de pescadores de comunidades ribeirinhas às Infecções Sexualmente Transmissíveis. Revista Cubana de Enfermería, 33(3). Recuperado de: http://www.revenfermeria.sld.cu/index.php/ enf/article/view/1231/288.

Sá, M. I., Silva, M. T., Almeida, D., Vieira, B., Lima, T., Conde, C., Teixeira, M., Lima, J., \& Oliveira, T. (2015). Infeções sexualmente transmissíveis e factores de risco nas adolescentes e jovens: Dados de um Centro de Atendimento a Jovens. Nascer e Crescer, 24(2), 64-69. Recuperado de http://www.scielo.mec.pt/scielo.php?scrip$\mathrm{t}=$ sci_arttext\&pid=S0872-07542015000300003\&Ing=pt\&tIng=pt. 
Sampaio, J., Santos, R. C. D., Callou, J. L. L., \& Souza, B. B. C. (2011). Ele não quer com camisinha e eu quero me prevenir: exposição de adolescentes do sexo feminino às IST/aids no semi-árido nordestino. Saude soc., 20(1), 171-181. Recuperado de: https://www.scielosp.org/article/sausoc/2011.v20n1/171-181/pt/.

Santos, C. M. A., Oliveira, J. D. S., Lima, S. V. M. A., Santos, A. D., Góes, M. A. O., \& Sousa, L. B. (2018). Conhecimentos, atitudes e prática de homens sobre infecções sexualmente transmissíveis. Cogitare Enfermagem, 23(1). http://dx.doi. org/10.5380/ce.v23i1.54101

Santos, C. P., Barboza, E. C. S., Freitas, N. O., Almeida, J. C., Dias, A. C., \& Araújo, E. C. (2016). Adesão ao uso do preservativo masculino por adolescentes escolares. Revista Brasileira de Pesquisa em Saúde/Brazilian Journal of Health Research, 18(2), 60-70. https://doi.org/10.21722/rbps.v18i2.15085

Sehnem, G. D., Pedro, E. N. R., Ressel, L. B., \& Vasquez, M. E. D. (2018). Adolescentes que vivem com HIV/aids: experiências de sexualidade. Revista Gaúcha de Enfermagem, 39, 1-22. http://dx.doi.org/10.1590/1983-1447.2018.2017-0194

Silva, A. T., Jacob, M. H. V. M., \& Hirdes, A. (2017). Conhecimento de adolescentes do ensino médio sobre DST/AIDS no sul do Brasil. Aletheia, (46), 34-49. Recuperado de: http://www.periodicos.ulbra.br/index.php/aletheia/article/view/3230/2379.

Silva, J., Oliveira, D., Rocha Filho, D., Mesquita, N., Lima, M., Teixeira, H., \& Costa, E. (2018). Vulnerabilidade às infecções sexualmente transmissíveis/ aids em idosos. Revista Uningá, 53(1). Recuperado de http://revista.uninga.br/index.php/uninga/ article/view/1418.

Silva, L. M. D., \& Cortez, E. A. (2017). Oficinas de educação permanente sobre infecção sexualmente transmissível: relato de experiência. Rev. enferm. UFPE on line, 11(supl. 5), 2250-2256. http://dx.doi.org/10.5205/reuol.9302-81402-1RV.1105sup201734

Sousa, L. M. S. D., Silva, L. S., \& Palmeira, A. T. (2014). Representações sociais de caminhoneiros de rota curta sobre HIV/AIDS. Psicologia \& Sociedade, 26(2), 346-355. https://dx.doi.org/10.1590/S0102-71822014000200011

Team, R. (2013). R development core team. RA Lang Environ Stat Comput, 55, 275-286. Recuperado de: http://citeseerx.ist.psu.edu/viewdoc/download?doi=10.1.1.470.5851\&rep=rep1\&type $=$ pdf

Teva, I., Bermúdez, M. P., \& Ramiro, M. T. (2013). Satisfacción sexual y actitudes hacia el uso del preservativo en adolescentes: evaluación y análisis de su relación con el uso del preservativo. Revista Latinoamericana de Psicología, 46(2), 127-136. https://doi.org/10.1016/S0120-0534(14)70016-0

Thiesen, F. (2018). Ist'se uso de preservativos. Revista UNIPLAC, 6(1). Recuperado de: https://revista.uniplac.net/ojs/index.php/uniplac/article/view/3627.

Vergès, P. (1992). L'évocation de l'argent: une méthode pour la définition du noyau central de la représentation. Bulletin de Psychologie, 45, 203-209. Recuperado de: http://psycnet.apa.org/record/1998-01851-006

Wachelke, J., \& Wolter, R. (2011). Critérios de construção e relato da análise prototípica para representações sociais. Psicologia: Teoria e Pesquisa, 27(4), 521-526. http://dx.doi.org/10.1590/S0102-37722011000400017 


\section{ANEXO. \\ Questionário}

Instruções: A pesquisa em questão trata-se de um estudo científico. Desta forma, garantimos aos colaboradores que estes dados serão tratados estatisticamente, bem como o anonimato e a confidencialidade dos dados será resguardada. Por favor, escreva as primeiras cinco palavras que lhe vierem à cabeça quando eu lhe digo as seguintes palavras.

Estímulo I (Infecções Sexualmente Transmissíveis)

Dados sociodemográficos

Idade: Anos Sexo: ( )Masculino ( )Feminino

Segue alguma religião? ( ) Sim. Qual? . ( ) Não

Você tem amizade ou conhece alguém que tenha alguma Infecção Sexualmente Transmissível? 